\title{
As contribuições da equipe multiprofissional da atenção básica de saúde frente aos
}

\section{cuidados paliativos}

The contributions of the multiprofessional team in basic health care in front of palliative care

Las contribuciones del equipo multiprofesional de atención básica de salud frente a la atención paliativa

Mariana Pereira Barbosa Silva

ORCID: https://orcid.org/0000-0003-0852-8099

Universidade Estadual do Piauí, Brasil

E-mail: marianapbsilvaa@gmail.com

Rayssa Stéfani Sousa Alves

ORCID: https://orcid.org/0000-0002-9666-675X

Pontifícia Universidade Católica de Goiás, Brasil

E-mail: rayssastefani02@gmail.com

Airton César Leite

ORCID: https://orcid.org/0000-0001-7184-8488

Centro Universitário Santo Agostinho, Brasil

E-mail: ainton.cesar2014@gmail.com

Stephany da Conceição Menezes

ORCID: https://orcid.org/0000-0002-8143-4366 Centro Universitário Mauricio de Nassau, Brasil

E-mail: stephanyfacul@gmail.com

Jamelyo Amancio Teodoro

ORCID: https://orcid.org/0000-0002-0815-7059

Centro Universitário de Caratinga, Brasil

E-mail: amancioteodoro97@gmail.com

Daniel Felipe da Silva Sales

ORCID: https://orcid.org/0000-0001-6827-2393 Universidade Potiguar, Brasil

E-mail: daniellfelipe.com@gmail.com

Nathália Caroline Flor dos Santos

ORCID: https://orcid.org/0000-0001-8980-5785 Universidade Potiguar, Brasil

E-mail: ncaroline09@hotmail.com

Laise Pereira da Silva Brito

ORCID: https://orcid.org/0000-0003-4326-0809

Universidade Estadual do Piauí, Brasil

E-mail: laisepsbrito@gmail.com

Danielle de Brito Rodrigues

ORCID: https://orcid.org/0000-0001-9898-2089

Centro Universitário Santo Agostinho, Brasil E-mail: danyeh97@gmail.com

Bruno Lopes Pereira

ORCID: https://orcid.org/0000-0003-2324-4721

Instituto Educacional Santo Agostinho, Brasil E-mail: brunoloperz192@gmail.com

Karina Aparecida Resende

ORCID: https://orcid.org/0000-0001-7812-7042

Universidade Federal de São João Del-Rei, Brasil

E-mail: karinaresendeufsj@gmail.com

Lucília da Costa Silva

ORCID: https://orcid.org/0000-0001-9386-5684

Centro Universitário Santo Agostinho, Brasil

E-mail: luciliafiso@outlook.com

Brena Carolina Andrade Bordalo Sampaio

ORCID: https://orcid.org/0000-0002-3551-8366

Escola Superior da Amazônia, Brasil

E-mail: brenabsampaio@gmail.com

Kelly Savana Minaré Baldo Sucupira

ORCID: https://orcid.org/0000-0003-1932-9458

Universidade Federal do Triângulo Mineiro, Brasil E-mail: kellyminare@gmail.com 


\title{
Resumo
}

Objetivo: Compreender qual o papel dos profissionais de saúde da Atenção Primaria à Saúde frente aos cuidados paliativos? Método: O estudo trata-se, de uma revisão integrativa de literatura, com abordagem qualitativa, realizada por meio de revisão de artigos publicados em base de dados da Biblioteca Virtual da Saúde (BVS). Foram utilizados os seguintes descritores em base DeCS (Descritores em Ciências da Saúde): Cuidados Paliativos; Cuidados de Enfermagem; Atenção Primária a Saúde. Durante as buscas, foram encontrados 21 artigos, destes, 08 integraram ao estudo. Os critérios de inclusão foram pesquisas bibliográficas publicadas entre os anos de 2002 a 2021 . Os critérios de exclusão foram artigos que não tivessem relevância a com temática do estudo. Resultados: Entende-se por qualidade de vida a possibilidade de autonomia em relação à tomada de decisões, bem como a minimização da dor e/ou de procedimentos invasivos que não possam modificar o processo de morrer com dignidade. Tendo em vista que os princípios dos cuidados paliativos são a oferta da qualidade de vida e controle da dor e sofrimento, compreende-se que a equipe multidisciplinar da Atenção Primaria à Saúde, precisa estar capacitada para proporcionar o atendimento paliativo humanizado no ambiente domiciliar do paciente. Conclusão: O presente alcançou com êxito os objetivos estabelecidos quanto as contribuições da equipe multidisciplinar da Atenção Primária à Saúde acerca do Cuidado Paliativo visando melhor qualidade assistencial e conforto ao paciente em sua fase terminal. Sendo assim, torna-se cada vez mais urgente a priorização dos cuidados paliativos pela Atenção Primaria à Saúde, possibilitando a organização, coordenação e assistência humanizada ao paciente em seu leito domiciliar.

Palavras-chave: Cuidados paliativos; Cuidados de enfermagem; Atenção primária a saúde.

\begin{abstract}
Objective: To understand the role of health professionals in Primary Health Care in relation to palliative care? Method: The study is an integrative literature review, with a qualitative approach, carried out by reviewing articles published in the database of the Virtual Health Library (VHL). The following descriptors were used on the basis of DeCS (Health Sciences Descriptors): Palliative Care; Nursing care; Primary Health Care. During the searches, 21 articles were found, of these, 8 were part of the study. The inclusion criteria were bibliographic searches published between the years 2002 to 2021. The exclusion criteria were articles that were not relevant to the theme of the study. Results: Quality of life is understood as the possibility of autonomy in relation to decision-making, as well as the minimization of pain and / or invasive procedures that cannot modify the process of dying with dignity. Bearing in mind that the principles of palliative care are the offer of quality of life and control of pain and suffering, it is understood that the multidisciplinary
\end{abstract}


team of Primary Health Care needs to be trained to provide humanized palliative care in the patient's home environment. Conclusion: The present has successfully achieved the objectives set regarding the contributions of the multidisciplinary team of Primary Health Care about Palliative Care aiming at better quality of care and comfort to the terminally ill patient. Thus, it becomes increasingly urgent to prioritize palliative care through Primary Health Care, enabling the organization, coordination and humanized assistance to the patient in their home bed.

Keywords: Palliative care; Nursing care; Primary health care.

\section{Resumen}

Objetivo: ¿Comprender el papel de los profesionales de la salud en la Atención Primaria de Salud en relación con los cuidados paliativos? Método: El estudio es una revisión integradora de la literatura, con enfoque cualitativo, realizada mediante la revisión de artículos publicados en la base de datos de la Biblioteca Virtual en Salud (BVS). Los siguientes descriptores se utilizaron sobre la base de DeCS (Descriptores de Ciencias de la Salud): Cuidados Paliativos; Cuidado de enfermera; Atención Primaria de Salud Durante las búsquedas se encontraron 21 artículos, de los cuales 8 formaban parte del estudio. Los criterios de inclusión fueron búsquedas bibliográficas publicadas entre los años 2002 a 2021 . Los criterios de exclusión fueron artículos que no fueran relevantes para la temática del estudio. Resultados: La calidad de vida se entiende como la posibilidad de autonomía en la toma de decisiones, así como la minimización del dolor y / o procedimientos invasivos que no pueden modificar el proceso de morir con dignidad. Teniendo en cuenta que los principios de los cuidados paliativos son la oferta de calidad de vida y el control del dolor y el sufrimiento, se entiende que el equipo multidisciplinar de Atención Primaria de Salud necesita estar capacitado para brindar cuidados paliativos humanizados en el entorno del hogar del paciente. Conclusión: El presente ha logrado con éxito los objetivos planteados en cuanto a las aportaciones del equipo multidisciplinario de Atención Primaria de Salud sobre Cuidados Paliativos con el objetivo de mejorar la calidad de la atención y el confort del paciente terminal. Así, se hace cada vez más urgente priorizar los cuidados paliativos a través de la Atención Primaria de Salud, posibilitando la organización, coordinación y asistencia humanizada al paciente en su cama de domicilio.

Palabras clave: Cuidados paliativos; Cuidado de enfermera; Primeros auxilios.

\section{Introdução}

Os cuidados paliativos correspondem a um tipo de abordagem terapêutica multiprofissional, que tem como base considerar o indivíduo como um todo, visando proporcionar cuidados e qualidade de vida ao indivíduo em situação de enfrentamento à enfermidade que ameaça a vida. Ou seja, ocorre a identificação do diagnóstico precoce, a fim de minimizar o sofrimento físico, psicológico, social e espiritual (Vargas et al., 2013; Gomes \& Othero, 2016).

Os cuidados paliativos tem como finalidade oferecer dignidade no momento da morte, tanto para o indivíduo quanto para os familiares, oferecendo-lhes acolhimento para enfrentar este momento de suas vidas. Sendo assim, as ações terapêuticas, além de auxiliarem no alívio dos sintomas, auxiliam na criação de uma atmosfera de cuidados acolhedora, designando uma rede de suporte que o acolha e ampare o enfermo e seus familiares neste momento de vulnerabilidade (Floriani \& Schramm, 2007).

Os cuidados paliativos são cuidados ativos e integrais concedido ao paciente cuja doença não responde mais ao tratamento curativo disponível. Conceitua - se, como uma abordagem de cuidados diferenciados, que não visa somente à prevenção e o alívio da dor e do sofrimento, mas melhorar a qualidade de vida do doente e seus familiares através da prevenção e identificação precoce de problemas não apenas físicos mas também psicossociais e espirituais (Araújo \& Silva, 2012).

De acordo com Gomes e Othero (2016), estes cuidados que são defendidos por profissionais paliativistas, têm como principal atribuição uma assistência integral e que proporcione uma morte digna ao paciente.

Nos anos 60, em uma atitude paternalista, o médico era o responsável por decidir qual conduta tomar quanto ao paciente que se encontrava em situação de prognostico de terminalidade, seja por doenças ou agravos, e apenas comunicava os familiares ou pessoas próximas do paciente sobre sua decisão (Franco et al., 2017). Ou seja, pacientes e familiares não tinham direito de questionar e acrescentar suas opiniões e possíveis decisões em relação ao passo seguinte na assistência executada.

Com o passar dos anos, a assistência paliativa se tornou discutível com familiares e paciente, desde o diagnóstico de uma doença terminal, até o processo de morte e morrer. O que se evidencia pela reafirmação dos Cuidados Paliativos da OMS, os tornando aplicáveis no tratamento inicial de doenças terminais, integrados ao tratamento curativo (Lanken et al., 2008). 
Tal afirmação declara que o diagnóstico de doenças agravantes deve ser realizado precocemente, para que o paciente possa ser avaliado, e a partir desta avaliação, possa ter uma assistência planejada, observando-o em todos os seus aspectos, bem como físicos, psicossociais e espirituais (Franco et al., 2017).

Isto se deve ao fato de que uma doença causa diversas alterações no indivíduo desde o seu início, até o seu término, e ainda afeta o paciente em todos os seus aspectos. Ainda se discute também, um cuidado constante durante toda a vida do paciente, quando este possui determinada doença crônico-degenerativa (Franco et al., 2017).

O conceito inicial de cuidados paliativos, adotado na década de 1960, visava aprimorar a qualidade de vida dos doentes com câncer em fase terminal. Entretanto, aos poucos esta percepção foi se estendendo à outras doenças de diversas características, capazes de resultar na fase terminal da vida (Chaves et al., 2011).

Segundo os estudos de Mechelen et al. (2012), os cuidados paliativos são voltados para indivíduos que enfrentam a luta contra o câncer e falência de órgãos.

Portanto, os cuidados paliativos destinam-se, não somente às neoplasias, mas a outras doenças crônico-degenerativas, tais como as demências, a doença de Parkinson, a insuficiência cardíaca, a insuficiência renal, a doença pulmonar obstrutiva crônica, dentre outras doenças em que não há perspectiva de cura (Fonseca \& Geovanini, 2013).

O cuidado a pacientes em fase final das suas vidas é, cada vez mais, uma realidade no cotidiano dos profissionais de saúde nos vários níveis de assistência. Isso se deve ao progressivo envelhecimento da população, e à maior sobrevida de portadores de doenças graves e fatais com evolução progressiva (Schneider et al., 2010; Florianí \& Schramm, 2007).

A Atenção Primária à Saúde compreende o nível primário responsável pela Atenção à Saúde da população. Além de oferecer fácil acesso ao sistema de saúde para todas as necessidades de seus usuários, os profissionais atuantes em unidades de Atenção Primária à Saúde acompanha suas histórias de vida, oferecendo atenção e cuidado integral, coordenando e integrando o atendimento prestado por outros serviços de saúde. Também compartilha algumas características com os outros níveis de assistência como atenção à prevenção, tratamento e reabilitação, assim como o trabalho em equipe (Starlfield, 2002).

Ainda que não existam no Brasil parâmetros oficiais para o cálculo das necessidades de Cuidados Paliativos, a Organização Mundial de Saúde afirma que essa necessidade pode ser estimada a partir do perfil de mortalidade de uma população, pois, entre as mortes ocorridas por causa natural, $50 \%$ a $80 \%$ seriam passíveis de Cuidados Paliativos, considerando-se todos os diagnósticos (Academia Nacional de Cuidados Paliativos, 2009).

Apesar da grande demanda e da complexidadedos casos, que muitas vezes requerem ações, procedimentos e tecnologias que não são usuais nas Unidades Básicas de Saúde, ainda são raros os estudos sobre quais ações caberiam aos profissionais da Atenção Primária à Saúde em cuidados paliativos.

Sendo assim, este estudo tem como objetivo compreender qual o papel dos profissionais de saúde da Atenção Primaria à Saúde frente aos cuidados paliativos?

\section{Metodologia}

O estudo trata-se, de uma revisão integrativa de literatura, com abordagem qualitativa.

A revisão integrativa é um método que tem como finalidade oferecer suporte para a tomada de decisão e a melhoria da prática clínica (Benefield, 2003), possibilitando a síntese do estado do conhecimento de um determinado assunto, além de apontar lacunas do conhecimento que precisam ser preenchidas com a realização de novos estudos. Este método de pesquisa permite a síntese de múltiplos estudos publicados e possibilita conclusões gerais a respeito de uma particular área de estudo (Polit e Beck, 2006).

A revisão integrativa da literatura consiste na construção de uma análise ampla da literatura, contribuindo para discussões sobre métodos e resultados de pesquisas, assim como reflexões sobre a realização de futuros estudos. O propósito 
inicial deste método de pesquisa é obter um profundo entendimento de um determinado fenômeno baseando-se em estudos anteriores (Broome, 2000).

É necessário seguir padrões de rigor metodológico, clareza na apresentação dos resultados, de forma que o leitor consiga identificar as características reais dos estudos incluídos na revisão (Beyea \& Nicoll, 1998).

Segundo Denzin e Lincoln (2006), a pesquisa qualitativa envolve uma abordagem interpretativa do mundo, o que significa que seus pesquisadores estudam as coisas em seus cenários naturais, tentando entender os fenômenos em termos dos significados que as pessoas a eles conferem.

Seguindo essa linha de raciocínio, Vieira e Zouain (2005) afirmam que a pesquisa qualitativa atribui importância fundamental aos depoimentos dos atores sociais envolvidos, aos discursos e aos significados transmitidos por eles. Neste sentido, esses autores afirmam que, esse tipo de pesquisa preza pela descrição detalhada dos fenômenos e dos elementos que o envolvem.

A pesquisa qualitativa tem o ambiente natural como sua fonte direta de dados e o pesquisador como seu principal instrumento; os dados coletados são predominantemente descritivos; a preocupação com o processo é muito maior do que com o produto; o significado que as pessoas dão às coisas e à sua vida são focos de atenção especial pelo pesquisador; e a análise dos dados tende a seguir um processo indutivo (Ludke \& André, 1986).

O levantamento de conteúdo foi realizado entre setembro de 2020 a março de 2021, por meio da busca de artigos indexados nas seguintes bases de dados: Scielo (Scientific Eletronic Library Online), BVS (Biblioteca Virtual em Saúde), Google Scholar, PubMed (National Center for Biotechnology Information), Lilacs (Literatura Latino-Americana e do Caribe em Ciências da Saúde), Revistas de Enfermagem.

Foram utilizados os seguintes descritores em base DeCS (Descritores em Ciências da Saúde): Cuidados Paliativos; Cuidados de Enfermagem; Atenção Primária a Saúde, utilizado o operador booleano AND.

Os critérios de inclusão para a seleção dos conteúdos foram, artigos na linguagem portuguesa, inglesa e espanhola. Publicados na íntegra de acordo com a temática referente à revisão integrativa, documentos, regulamentações, normativas de entidades de saúde acerca do tema, artigos, teses, e dissertações publicados nos referidos bancos de dados compreendendo os anos de 2002 a 2021.

Os critérios de exclusão são estudos mediante a recompensação monetária, e materiais que não possuem relevância com a temática proposta.

\section{Resultados e Discussão}

A partir da revisão de literatura e análise dos estudos indexados nas bases de dados eletrônicas, acerca da temática proposta, foram encontrados 21 estudos científicos, sendo que, apenas 16 estudos foram selecionados, 12 atenderam aos critérios de inclusão previamente estabelecidos, destes, 04 foram excluídos com base nos critérios de exclusão, restando 08 artigos para composição e análise do estudo. O fluxograma com o detalhamento das etapas de pesquisa está apresentado a seguir na Figura 1. 
Figura 1. Fluxograma de identificação e seleção dos artigos. 2021.

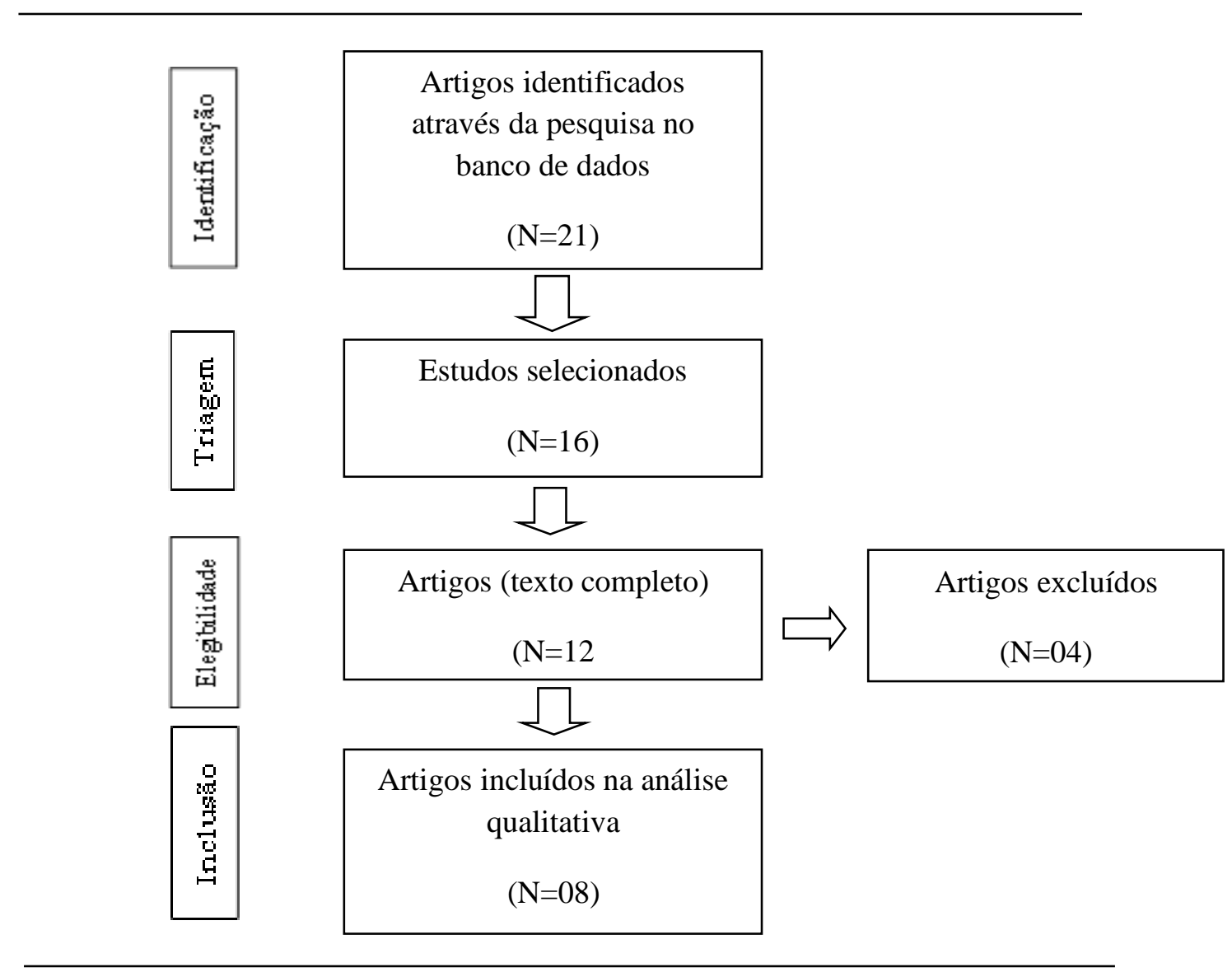

Fonte: Elaboração própria (2021).

O presente estudo trouxe a oportunidade de conhecer de uma forma mais aprofundada os cuidados paliativos, sua origem e princípios, bem como os pontos positivos e negativos dessa linha de cuidados. A pesquisa foi satisfatória e alcançou com êxito os objetivos estabelecidos quanto as contribuições da equipe multidisciplinar da Atenção Básica de Saúde acerca do Cuidado Paliativo visando melhor qualidade assistencial e conforto ao paciente em sua fase terminal.

Os cuidados paliativos estão plenamente inseridos no âmbito da reflexão bioética, especialmente no que se refere ao processo de tomada de decisões envolvendo questões relacionadas aos Cuidados Paliativos na Atenção Primária à Saúde (Vieira \& Goldim, 2012).

Segundo os estudos de Cardoso et al. (2013), os Cuidados Paliativos compreende todas as condições do paciente, portanto, em relação aos sintomas físicos, tem se uma necessidade maior de uma equipe multiprofissional, visando consenso no método terapêutico empregado, para que a assistência paliativa não se torne de alguma forma distanásia, trazendo sofrimento longo ao paciente, ou se aproxime da eutanásia, acelerando o processo de morte.

Durante o processo de morte, uma das intervenções dos Cuidados Paliativos consiste na administração de medicações fortes, por exemplo, os opioides utilizados para o alivio da dor. Portando, existe uma alta porcentagem de recusa dos pacientes devido ao grau de sedação que a medicação provoca no indivíduo ao ser administrada. Sendo assim, deve - se respeitar a decisão do paciente, em passar pela morte de forma lúcida, porém sem o alívio da dor (Baliza et al., 2012).

Em relação ao processo de morte e morrer, pode ser afirmado que os pacientes que entram em protocolo de Cuidados Paliativos são pacientes em sua grande maioria, que permaneceram por um longo período em tratamento com a equipe, onde os 
profissionais depositaram seus esforços e criaram vínculos mesmo que simples, e em seguida os mesmos pacientes vão a óbito (Almeida et al., 2014).

Os profissionais de saúde inseridos nos Cuidados Paliativos, frente ao processo de Morte e Morrer, participa diariamente do sofrimento destes pacientes e de seus familiares na espera da morte, até que ela chegue. Sendo assim, esses eventos tornamse cotidiano de suas profissões, portanto, na maioria das vezes, os profissionais não sabem como lidar estas situações (Silva et al., 2015; Sales \& Alencastre, 2003).

Posto isso, não saber como lidar frente o processo de morte e morrer é um evento comum entre os profissionais de saúde, onde já não conseguem enxergar a morte como um acontecimento natural. Assim, evidencia a necessidade do desenvolvimento de estratégias para que essas situações não afete os profissionais em um longo prazo (Silva et al., 2015; Sales \& Alencastre, 2003).

Em alguns momentos, os profissionais de saúde podem se sentir impotente, visto que, não pode fazer mais nada por um paciente, bem como, sensação de despreparo profissional, visto que, apenas aprendeu a curar. A morte eminente pode fazer com que estes sentimentos primários se transformem, em raiva, frustração, visto que muitos materializam o evento que era natural, como de sua responsabilidade, como se pudessem ter feito algo mais, ou evitado alguma atitude. A morte passa a ser incômoda de alguma forma, os fazendo inclusive negociar, ou manifestar comportamentos defensivos em alguns casos (Germano \& Meneguin, 2013).

Santos et al. (2017) afirma em seus estudos que, em relação ao respeito, e a decisão por não promover o adiantamento da morte, percebe-se que existe uma falta de conhecimento do que realmente são os Cuidados Paliativos no processo de Morte e Morrer, sendo comumente confundidos com cuidados críticos, a diferença está na essência da paliativação, onde já não existe a cura de alguma condição de saúde como objetivo.

Segundo Castro et al. (2016), quanto à promoção de suporte psicossocial e espiritual, pode-se, afirmar que esta é uma das dimensões do cuidar que mesmo quando não se trata de Cuidados Paliativos, ainda é pouco aplicada à prática hospitalar. Esta dificuldade pode estar relacionada com sua forma atual, que é abstrata para os profissionais, sendo difícil identificar, diagnosticar, prescrever cuidados, executá-los e ainda avaliar seus resultados.

O profissional que atua nos Cuidados Paliativos, age como um solucionador, ou seja, tem por papel avaliar toda e qualquer necessidade não suprida, e propor soluções para elas. As necessidades psicossociais e espirituais não deixem de ser uma delas, então devem ser propostos e executados suportes para estas (Germano \& Meneguin, 2013).

A espiritualidade permite que o paciente e todos aqueles envolvidos em sua rotina, familiares, profissionais, encontrem sua unidade, tendo uma noção muito mais ampla sobre a vida e o seu papel nela, repensando os valores que cercam situações como a morte eminente, e encontrando um sentido natural e pleno para que esse tipo de situação com toda e qualquer pessoa (Germano \& Meneguin, 2013).

Quanto à promoção de autonomia e independência, é importante que os profissionais de saúde compreenda o conceito dos cuidados paliativos para que sua posição profissional não interfira na decisão do paciente e seus familiares quanto a autonomia de exercerem seus direitos sobre a terapêutica a ser empregada (Gulini et al., 2017).

Já quanto à independência, uma das medidas que promove a independência, assim como autonomia do paciente, é o acompanhamento paliativo desde o diagnóstico de uma doença degenerativa, ou o suporte a saúde de forma mais precoce possível, nestas situações. Isto permite que o paciente enfrente menos danos em longo prazo. Porém, atualmente, as equipes de saúde têm enxergado os Cuidados Paliativos apenas como aqueles que são executados nos últimos dias de vida de um paciente, o que acarreta em um modelo de assistência preventivo "inexistente" (Gulini et al., 2017).

O acompanhamento multidisciplinar permite também um foco maior em todas as necessidades do paciente, favorecendo uma abordagem mais humanizada e que permita maior independência ao paciente, uma vez que o foco se torna cada vez menos 
a patologia ou o agravo de saúde. Isto gera um atendimento diferenciado, com profissionais que identificaram e auxiliaram na perda de independência progressiva (Matos, Pires \& Sousa, 2010).

Em relação ao fornecimento de assistência aos familiares e pessoas próximas ao paciente, a equipe de saúde deve promover a participação familiar em toda a terapêutica, incluindo estas pessoas no âmbito hospitalar do paciente, e também deve fazer com que o paciente se enxergue como corresponsável, junto de seus entes, mas também como protagonistas, tendo papel ativo em todas as decisões e atitudes da equipe de saúde (Silva et al., 2015).

A Organização Mundial da Saúde (OMS), desde 2002, define Cuidado Paliativo como uma abordagem que promove a qualidade de vida de pacientes e seus familiares que enfrentam doenças que ameacem a continuidade da vida, por meio da prevenção e do alívio do sofrimento. Requer identificação precoce, avaliação e tratamento da dor e outros problemas de natureza física, psicossocial e espiritual (OMS, 2011).

Não é possível relatar, mesmo que sucintamente, todas as necessidades de cuidados dessas pessoas e suas famílias, pois antes de tudo, é fundamental se ter em mente que cada um é produto de sua história de vida, valores, crenças, condiçãosocial e acesso à saúde. Desse modo, é fundamental que todos os envolvidos nessa tarefa enfrentem tal complexidade sem receios, para prestarem assistência integral a esses pacientes e seus familiares, nesse momento de maior fragilidade (Conselho Regional de Medicina do Estado de São Paulo, 2008).

Ainda assim, pacientes considerados fora de possibilidade de cura acumulam-se nos hospitais, recebendo muitas vezes assistência inadequada, ainda focada na tentativa de "salvar a vida", utilizando métodos invasivos e tecnologias duras. Essa abordagem ignora o sofrimento, não trata os sintomas mais prevalentes e frequentemente mantém o indivíduo isolado desua família, de seu lar e de suas lembranças (Academia Nacional de Cuidados Paliativos, 2009).

A morte ocorre solitária. Não se trata de incentivar uma atitude contrária à medicina tecnológica, mas questionar a centralidade do "curar" e o uso de tecnologias duras na prática e mesmo na formação dos profissionais de saúde. É, antes, um incentivo à reflexão sobre a conduta dos profissionais de saúde diante da finitude humana (Academia Nacional de Cuidados Paliativos, 2009).

No Brasil, ainda são poucos os serviços de cuidados paliativos, apesar de já estar promulgada legislação específica estabelecendo no âmbito do Sistema Único de Saúde (SUS) o cuidado paliativo e o atendimento/internação domiciliar (Brasil, 2002).

Observa-se o crescimento da oferta desses serviços ano a ano, ainda que a maioria desses esteja ligada a hospitais especializados e de ensino, por meio de internação, atendimento ambulatorial e domiciliar (Conselho Regional de Medicina do Estado de São Paulo, 2008; Rabelo \& Rodrigues, 2010).

Em vários países, entretanto, a Atenção Primária à Saúde (APS) é considerada o melhor nível de assistência à saúde paraa prestação e coordenação dos cuidados paliativos de seus usuários em seu ambiente domiciliar (Borgsteede et al., 2007; Barnes et al., 2007).

Isso porque se entende que a proximidade geográfica, cultural e emocional desses profissionais pode contribuir em muito para que o cuidado ocorra de forma humanizada, respeitando-se a autonomia de pacientes e famílias de forma coordenada, evitando-se a fragmentação do indivíduo a partirdos múltiplos especialistas que usualmente estão envolvidos nesses casos, devido à sua complexidade (McDaniel, 2005).

Além disso, a Atenção Primaria à Saúde pode estruturar-se de modo a privilegiar a permanência do paciente em cuidados paliativos no domicílio, evitando seu afastamento da família em seus momentos finais de vida (Florianí \& Schramm, 2007).

Os profissionais de saúde devem estar atentos às necessidades psicológicas da família, visto que a morte é um evento que traz sentimentos intensos como a raiva, frustração, luto, que podem desencadear em danos maiores em longo prazo. Devem estar aptos para identificar sinais de comportamento alterado e oferecer apoio e suporte da equipe multidisciplinar, ajudando a família a passar por esta fase da vida. Tanto no contato com a família, quanto no contato com o paciente, existe uma necessidade de conhecimento de técnicas e estratégias de comunicação interpessoal, sejam verbais ou não verbais, a fim de trazer mais 
clarividência a tudo àquilo que a equipe de saúde deseja passar, seja para transmitir sentimentos afetuosos, comunicar procedimentos, explicar a terapêutica empregada (Silva \& Araújo, 2012).

\section{Considerações Finais}

O presente estudo trouxe a oportunidade de compreender os cuidados paliativos de forma mais aprofundada, bem como, sua origem, seus princípios, pontos positivos e negativos dessa linha de cuidado pouco efetiva na Atenção Primaria à Saúde.

A pesquisa foi satisfatória e alcançou com êxito os objetivos estabelecidos quanto as contribuições da equipe multidisciplinar da Atenção Básica de Saúde acerca dos Cuidados Paliativos visando uma melhor qualidade assistencial e conforto ao paciente em sua fase terminal da vida.

Sendo assim, torna-se cada vez mais urgente a priorização dos cuidados paliativos pela Atenção Primaria à Saúde, possibilitando a organização, coordenação e assistência humanizada ao paciente em seu leito domiciliar.

Os resultados do estudo enfatiza que, uma importante contribuição dos profissionais da Atenção Primária à Saúde para aprimorar a qualidade e o conforto do paciente inserido na assistência paliativa, consiste em proporcionar o atendimento humanizado domiciliar, possibilitando que o momento da morte não seja um momento solitário, longe de suas lembranças familiares.

Assim, torna - se, necessário a efetivação dessa estratégia quando não há mais possibilidades de cura para as condições de saúde do indivíduo, pois mesmo quando não houver mais cura, sempre haverá cuidados que visem a integridade, bem estar e o conforto do paciente em seus últimos dias de vida.

\section{Referências}

Academia Nacional de Cuidados Paliativos (ANCP). (2009). Manual de cuidados paliativos. Diagraphic.

Almeida, C. S. L., Sales, C. A., \& Marcon, S. S. (2014). O existir da enfermagem cuidando na terminalidade da vida: um estudo fenomenológico. Rev Esc Enferm USP.48(1):34-40.

Araújo, M. M. T., \& Silva, M. J. P. (2012). O conhecimento de estratégias de comunicação no atendimento à dimensão emocional em cuidados paliativos. Texto contexto - enferm. 21(1): 121-9.

Baliza, M. F., Bousso, R. S., Spineli, V. M. C. D., Silva, L., \& Poles, K. (2012). Cuidados paliativos no domicílio: percepção de enfermeiras da Estratégia Saúde da Família. Acta Paul Enferm.25(2):13-8.

Barnes, E. A., Fan, G., Harris, K., Barbera, L., Tsao, M., Doyle, M., et al. (2007). Involvement of Family Physicians in the Care of Cancer Patients Seen in the Palliative Rapid Response Radiotherapy Program. J Clin Oncol. 25(36): 5758-62.

Borgsteede, S. D., Deliens, L., Wal, G. V. D., Francke, A. L., Stalman, W. A. B., \& Eijk, J. T. M. E. (2007). Interdisciplinary cooperation of GPs in palliative care at home: A nationwide survey in the Netherlands. Scand J Prim Health Care. 25: 226-31.

Brasil. Lei $\mathrm{n}^{\circ}$ 10.424, de 15 de abril de 2002. (2002). Acrescenta capítulo e artigo à Lei ${ }^{\circ}$ 8.080, de 19 de setembro de 1990 , que dispõe sobre as condiçõespara a promoção, proteção e recuperação da saúde, a organização e o funcionamento de serviços correspondentes e dá outras providências, regulamentando a assistência domiciliar no Sistema Único de Saúde. Diário Oficial da República Federativa do Brasil; Abr 2002.

Cardoso, D. H., Muniz, R. M., Schwartz, E., \& Arrieira, I. C. O. (2013). Cuidados paliativos na assistência hospitalar: a vivência de uma equipe multiprofissional. Texto Contexto Enferm.22(4):1134-41.

Castro, M. C., Fuly, P. S., Garcia, T. R., \& Santos, M. L. (2016). Subconjunto terminológico CIPE® para pacientes em cuidados paliativos com feridas tumorais malignas. Acta Paul Enferm. 29(3):340-6.

Conselho Regional de Medicina do Estado de São Paulo (CREMESP). (2008). Cuidado Paliativo. CREMESP.

Floriani, C. A., \& Schramm, F. R. (2007). Desafios morais e operacionais da inclusão dos cuidados paliativos na rede de atenção básica. Cad. Saúde Pública. Sep; 23(9): 2072- 80.

Germano, K. S., \& Meneguin, S. (2013). Significados atribuídos por graduandos de enfermagem aos cuidados paliativos. Acta Paul Enferm.26(6):522-8.

Gomes, A. L. Z., \& Othero, M. B. (2016). Cuidados Paliativos. Estud. av.30(88):155-66.

Gulini, J. E. H. M. B., Nascimento, E. R. P., Moritz, R. D., Rosa, L. M., Silveira, N. R., \& Vargas, M. A. O. (2017). A equipe da Unidade de Terapia Intensiva frente ao cuidado paliativo: discurso do sujeito coletivo. Rev Esc Enferm USP.51:e03221. 
Research, Society and Development, v. 10, n. 4, e40210413887, 2021

(CC BY 4.0) | ISSN 2525-3409 | DOI: http://dx.doi.org/10.33448/rsd-v10i4.13887

Hermes, H. R., \& Lamarca, I. C. A. (2013). Cuidados paliativos: uma abordagem a partir das categorias profissionais de saúde. Ciênc Saúde Coletiva. Sep. 18(9):2577-88.

McDaniel, S. H., Campbell, Th. L., Hepworth, J., \& Lorenz, A. (2005). Family-Oriented Primary Care. Springer.

Mechelen, W. V., Aertgeerts, B., Ceulaer, K., Thoonsen, B., Vermandere, M., Warmenhoven, F., et al. (2012). Defining the palliative care patieny: A systematic review. Palliative Med. Feb; 27(2):197-208.

Ministério da Saúde. (2013). Cadernos da Atenção Básica. No 13, Controle dos cânceres do colo do útero e da mama. (2a ed.), MS.

Rabelo, C. A. F. G., \& Rodrigues, P. H. A. (2010). Saúde da Família e cuidados paliativos infantis: ouvindo os familiares de crianças dependentes de tecnologia. CiêncSaúde Coletiva. 15(Supl. 2): 3157-66.

Sales, C. A., \& Alencastre, M. B. (2003). Cuidados paliativos: uma perspectiva de assistência integral à pessoa com neoplasia. Rev Bras Enferm.56(5):566-569.

Santos, D. C., Silva, M. M., Moreira, M. C., Zepeda, K. G., \& Gaspar, R. B. (2017). Planejamento da assistência ao paciente em cuidados paliativos na terapia intensiva oncológica. Acta Paul Enferm. 30(3):295-300

Schneider, N., Lueckmann, S. L., Kuehne, S. L. F., Klindtworth, K., \& Behmann, M. (2010). Developing targets for public health initiatives to improve palliative care. BMC Public Health.10:222.

Silva, A. F., Issi, H. B., Motta, M. G. C., \& Botene, D. Z. A. (2015). Cuidados paliativos em oncologia pediátrica: percepções, saberes e práticas na perspectiva da equipe multiprofissional. Rev Gaúcha Enferm.36(2):56- 62.

Silva, M. J. P., \& Araújo, M. M. T. (2012). Estratégias de comunicação utilizadas por profissionais de saúde na atenção à pacientes sob cuidados paliativos. Rev Esc Enferm USP.46(3):626-32.

Silva, R. C. F. D., \& Hortale, V. A. (2006). Cuidados paliativos oncológicos: elementos para o debate de diretrizes nesta área. Cad. Saúde Pública.22(10):205566.

Starlfield B. (2002). Atenção primária: equilíbrio entre necessidades de saúde, serviços e tecnologia. UNESCO, Ministério da Saúde.

Vargas, M. A. O., Vivan, J., Vieira, R. W., Mancia, J. R., Ramos, F. R. S., Ferrazzo, S., et al. (2013). Redefining palliative care at a specialized care center: a possible reality? Texto Contexto Enferm. 22(3):637-45.

Verhoeven, A. A. H., Schuling, J., \& Maeckelber, E. L. M. (2011). The death of a patient: a model for reflection in GP training. BMC Fam Pract. 12(2): 8-15.

Walshe, C., Todd, C., Caress, A. L., \& Graham, C. C. (2008). Judgements about fellow professionals and the management of patients. Br J Gen Pract. 58: 264-72.

World Health Organization. (2011). Palliative care for older people: better practices. WHO.

Worldwide Palliative Care Alliance. (2014). Global Atlas of palliative care at the end of life. WHO. 